\title{
SSDB spaces and maximal monotonicity
}

\author{
Stephen Simons
}

Received: 19 June 2010 / Accepted: 22 June 2010 / Published online: 4 July 2010

(C) The Author(s) 2010. This article is published with open access at Springerlink.com

\begin{abstract}
In this paper, we develop some of the theory of SSD spaces and SSDB spaces, and deduce some results on maximally monotone multifunctions on a reflexive Banach space.
\end{abstract}

Keywords Fenchel duality theorem - Attouch-Brezis theorem - Symmetric bilinear form . Fenchel conjugate Hilbert space · Reflexive Banach space - Monotone and maximally monotone sets and multifunctions - Rockafellar's surjectivity theorem - Sum theorem · Abstract Hammerstein theorem

\section{Introduction}

In this paper, we develop enough of the theory of SSD spaces and SSDB spaces that we can obtain some significant results on maximally monotone multifunctions on a reflexive Banach space. With a few minor additions, this is a written version of the lecture with the same title delivered at the IX ISORA meeting in Lima, Peru, in October 2009, and we will not attempt to give a comprehensive exposition of the theory of SSD spaces and SSDB spaces. For this, we refer the reader to [13] and [14], from which many of the proofs given here are taken.

Many of the original results on maximally monotone multifunctions on a reflexive Banach space were obtained using Brouwer's fixed-point theorem either directly or indirectly. The approach given here is based on convex analysis - more specifically the Fenchel duality theorem. In Sect. 2, we give the three versions of the Fenchel duality theorem that we will use in this paper.

In Sect. 3, we introduce the concepts of SSD space, q-positive set and the functions $\Phi$., which are the generalizations to SSD spaces of Fitzpatrick functions of monotone sets. We also introduce the $q$-positive sets $\mathcal{P}_{q}(\cdot)$ determined by certain convex functions. Despite the fact that this section uses the idea of conjugate function from convex analysis, the

S. Simons $(\bowtie)$

Department of Mathematics, University of California, Santa Barbara, CA 93106-3080, USA

e-mail: simons@math.ucsb.edu 
arguments used are essentially algebraic, apart from the disguised differentiability argument of Lemma 3.7.

In Sect. 4, we introduce the concept of SSDB space, which is a SSD space with an appropriate Banach space structure. The main results of this section are the two "pos-neg theorems", Theorems 4.3 and 4.6 and the criterion for and properties of maximal $q$-positivity contained in Theorem 4.4.

For the rest of this paper, we suppose that $E$ is a reflexive Banach space. In Sect. 5, we show how $E \times E^{*}$ can be considered as a SSDB space, and how Theorems 2.4 and 4.4 lead to results on maximal monotonicity. Theorems 5.1 and 5.4 will be used in Sect. 6 .

Up to this point, our discussion of monotonicity has been in terms of monotone subsets of $E \times E^{*}$. In Sect. 6, we move the emphasis to monotone multifunctions from $E$ into $E^{*}$. In Theorem 6.3, we show how Theorem 5.1 gives Rockafellar's surjectivity theorem, and in Theorem 6.5, we show how Theorem 5.4 gives sufficient conditions for the sum of maximally monotone multifunctions to be maximally monotone.

In the final section of this paper, Sect. 7, we show how the pos-neg theorem, Theorem 4.6, together with the simple properties of the reflection maps $\rho_{1}, \rho_{2}: E \times E^{*} \rightarrow E \times E^{*}$ defined by $\rho_{1}\left(x, x^{*}\right):=\left(-x, x^{*}\right)$ and $\rho_{2}\left(x, x^{*}\right):=\left(x,-x^{*}\right)$ lead to an abstract Hammerstein theorem.

The author would like to express his appreciation to Radu Ioan Bot for some very constructive comments on the first version of this paper, and to the two anonymous referees for their very constructive comments on the paper as submitted.

\section{Versions of the fenchel duality theorem}

All vector spaces in the paper will be real. If $X$ is a nonzero vector space and $f: F \mapsto$ ]$-\infty, \infty]$ then we write $\operatorname{dom} f:=\{x \in F: f(x) \in \mathbb{R}\}$. The set $\operatorname{dom} f$ is the effective domain of $f . f$ is said to be proper if $\operatorname{dom} f \neq \emptyset$. We write $\mathcal{P C}(X)$ for the set of proper convex functions from $X$ into $]-\infty, \infty]$. If $X$ is a nonzero normed space we write $\mathcal{P C} \mathcal{L S C}(X)$ for $\{f \in \mathcal{P C}(X): f$ is lower semicontinuous $\}$. If $E$ and $F$ are nonzero vector spaces, $\langle\cdot, \cdot\rangle: E \times F \rightarrow \mathbb{R}$ is a bilinear form and $f \in \mathcal{P C}(E)$ then the Fenchel conjugate, $f^{*}$, of $f$ with respect to $\langle\cdot, \cdot\rangle$ is defined for $y \in F$ by

$$
f^{*}(y):=\sup _{x \in E}[\langle x, y\rangle-f(x)] .
$$

The commonest (but not the only) use of this notation is when $E$ is a normed space, $F$ is the dual, $E^{*}$, of $E$ and $\langle\cdot, \cdot\rangle$ is the duality pairing.

We start off by stating a result that is an immediate consequence of Rockafellar's version of the Fenchel duality theorem (see [11], Theorem 1, pp. 82-83 for the original version and Zălinescu, [17], Theorem 2.8.7, pp. 126-127 for more general results):

Theorem 2.1 Let $F$ be a nonzero normed space, $f: F \mapsto]-\infty, \infty]$ be proper and convex, $g: F \mapsto \mathbb{R}$ be convex and continuous, and $f+g \geq 0$ on $F$. Then there exists $z^{*} \in F^{*}$ such that $f^{*}\left(z^{*}\right)+g^{*}\left(-z^{*}\right) \leq 0$.

Theorem 2.2 below was first proved by Attouch-Brezis (this follows from [1], Corollary 2.3, pp. 131-132 ) - there is a somewhat different proof in Simons, [13], Theorem 15.1, pp. 66, and a much more general result was established in Zălinescu, [17], Theorem 2.8.6, pp. 125-126. We note that the result contained in Simons, [13], Theorem 8.4, pp. 46 implies both Theorems 2.1 and 2.2, and that [13], Theorem 7.4, pp. 43 gives a sharp lower bound on the possible values of $\left\|z^{*}\right\|$. 
Theorem 2.2 Let $E$ be a nonzero Banach space, $f, g \in \mathcal{P C} \mathcal{L S C}(E), f+g \geq 0$ on $E$ and $\bigcup_{\lambda>0} \lambda[\operatorname{dom} f-\operatorname{dom} g]$ be a closed linear subspace of $E$. Then there exists $z^{*} \in E^{*}$ such that $f^{*}\left(z^{*}\right)+g^{*}\left(-z^{*}\right) \leq 0$.

Notation 2.3 If $E$ and $F$ are nonzero normed spaces, we define the dual of $E \times F$ to be $E^{*} \times F^{*}$ under the pairing $\left\langle(x, y),\left(x^{*}, y^{*}\right)\right\rangle:=\left\langle x, x^{*}\right\rangle+\left\langle y, y^{*}\right\rangle \quad\left((x, y) \in E \times F,\left(x^{*}, y^{*}\right)\right.$ $\left.\in E^{*} \times F^{*}\right)$. We then define the projection maps $\pi_{1}, \pi_{2}$ by $\pi_{1}(x, y):=x$ and $\pi_{2}(x, y):=y$.

We end this section with a bivariate generalization of Theorem 2.2, which was first proved in Simons-Zălinescu, [15], Theorem 4.2, pp. 9-10. There was a simpler proof given in Simons, [13], Theorem 16.4, pp. 68-69. The hypothesis of Theorem 2.4 is that $h(x, \cdot)$ is the "inf-convolution" of $f(x, \cdot)$ and $g(x, \cdot)$, and the conclusion is that $h^{*}\left(\cdot, y^{*}\right)$ is the "exact inf-convolution" of $f^{*}\left(\cdot, y^{*}\right)$ and $g^{*}\left(\cdot, y^{*}\right)$.

Theorem 2.4 Let $E$ and $F$ be nonzero Banach spaces, $f, g: E \times F \mapsto]-\infty, \infty]$ be proper, convex and lower semicontinuous, $\bigcup_{\lambda>0} \lambda\left[\pi_{1} \operatorname{dom} f-\pi_{1} \operatorname{dom} g\right]$ be a closed linear subspace of $E$ and, for all $(x, y) \in E \times F$,

$$
h(x, y):=\inf \{f(x, u)+g(x, v): u, v \in F, u+v=y\}>-\infty .
$$

Then, for all $\left(x^{*}, y^{*}\right) \in E^{*} \times F^{*}=(E \times F)^{*}$,

$$
h^{*}\left(x^{*}, y^{*}\right)=\min \left\{f^{*}\left(s^{*}, y^{*}\right)+g^{*}\left(t^{*}, y^{*}\right): s^{*}, t^{*} \in E^{*}, s^{*}+t^{*}=x^{*}\right\} .
$$

\section{SSD spaces}

Definition 3.1 We will say that $(B,\lfloor\cdot, \cdot\rfloor)$ is a symmetrically self-dual space (SSD space) if $B$ is a nonzero real vector space and $\lfloor\cdot, \cdot]: B \times B \rightarrow \mathbb{R}$ is a symmetric bilinear form. In this case, we will always write $q(b):=\frac{1}{2}\lfloor b, b\rfloor(b \in B)$. (" $q$ " stands for "quadratic".)

Now let $(B,\lfloor\cdot, \cdot\rfloor)$ be a SSD space and $A \subset B$. We say that $A$ is $q$-positive if $A \neq \varnothing$ and

$$
b, c \in A \Longrightarrow q(b-c) \geq 0 .
$$

In this case, since $q(0)=0$,

$$
b \in A \Longrightarrow \inf q(A-b)=0 .
$$

We then define $\left.\left.\Phi_{A}: B \rightarrow\right]-\infty, \infty\right]$ by

$$
\Phi_{A}(b):=\sup _{A}[\lfloor\cdot, b\rfloor-q] \quad(b \in B) .
$$

$\Phi_{A}$ is a generalization to SSD spaces of the "Fiztpatrick function" of a monotone set, which was originally introduced in [6] in 1988, but lay dormant until it was rediscovered by Martínez-Legaz and Théra in [8] in 2001. We note then that, for all $b \in B$,

$$
\left.\begin{array}{rl}
\Phi_{A}(b) & =q(b)-\inf _{a \in A}[q(a)-\lfloor a, b\rfloor+q(b)] \\
& =q(b)-\inf _{a \in A} q(a-b)=q(b)-\inf q(A-b) .
\end{array}\right\}
$$

From (1),

$$
\Phi_{A}=q \text { on } A .
$$

Thus $\Phi_{A} \in \mathcal{P C}(B)$. We say that $A$ is maximally $q$-positive if $A$ is $q$-positive and $A$ is not properly contained in any other $q$-positive set. In this case, if $b \in B$ and inf $q(A-b) \geq 0$ then 
clearly $b \in A$. In other words, $(b \in B \backslash A \Longrightarrow \inf q(A-b)<0)$. From (1), inf $q(A-b) \leq 0$ and (inf $q(A-b)=0 \Longleftrightarrow b \in A$ ). Thus, from (3)

$$
\Phi_{A} \geq q \text { on } B \text { and }\left(\Phi_{A}(b)=q(b) \Longleftrightarrow b \in A\right) .
$$

We make the elementary observation that if $b \in B$ and $q(b) \geq 0$ then the linear span $\mathbb{R} b$ of $\{b\}$ is $q$-positive.

We now give some examples of SSD spaces and their associated $q$-positive sets. These examples are taken from [13], pp. 79-80.

Example 3.2 Let $B$ be a Hilbert space with inner product $(b, c) \mapsto\langle b, c\rangle$.

(a) If, for all $b, c \in B,\lfloor b, c\rfloor:=\langle b, c\rangle$ then $q(b)=\frac{1}{2}\|b\|^{2}$ and every nonempty subset of $B$ is $q$-positive.

(b) If, for all $b, c \in B,\lfloor b, c\rfloor:=-\langle b, c\rangle$ then $q(b)=-\frac{1}{2}\|b\|^{2}$ and the $q$-positive sets are the singletons.

(c) If $B=\mathbb{R}^{3}$ and

$$
\left\lfloor\left(b_{1}, b_{2}, b_{3}\right),\left(c_{1}, c_{2}, c_{3}\right)\right\rfloor:=b_{1} c_{2}+b_{2} c_{1}+b_{3} c_{3},
$$

then $q\left(b_{1}, b_{2}, b_{3}\right)=b_{1} b_{2}+\frac{1}{2} b_{3}^{2}$. Here, if $M$ is any nonempty monotone subset of $\mathbb{R} \times \mathbb{R}$ (in the obvious sense) then $M \times \mathbb{R}$ is a $q$-positive subset of $B$. The set $\mathbb{R}(1,-1,2)$ is a $q$-positive subset of $B$ which is not contained in a set $M \times \mathbb{R}$ for any monotone subset of $\mathbb{R} \times \mathbb{R}$. The helix $\{(\cos \theta, \sin \theta, \theta): \theta \in \mathbb{R}\}$ is a $q$-positive subset of $B$, but if $0<\lambda<1$ then the helix $\{(\cos \theta, \sin \theta, \lambda \theta): \theta \in \mathbb{R}\}$ is not.

Example 3.3 Let $E$ be a nonzero Banach space and $B:=E \times E^{*}$. For all $\left(x, x^{*}\right)$ and $\left(y, y^{*}\right) \in B$, we set $\left\lfloor\left(x, x^{*}\right),\left(y, y^{*}\right)\right\rfloor:=\left\langle x, y^{*}\right\rangle+\left\langle y, x^{*}\right\rangle$. Then $(B,\lfloor\cdot, \cdot\rfloor)$ is a SSD space with $q\left(x, x^{*}\right)=\frac{1}{2}\left[\left\langle x, x^{*}\right\rangle+\left\langle x, x^{*}\right\rangle\right]=\left\langle x, x^{*}\right\rangle$. Consequently, if $\left(x, x^{*}\right),\left(y, y^{*}\right) \in B$ then $\left\langle x-y, x^{*}-y^{*}\right\rangle=q\left(x-y, x^{*}-y^{*}\right)=q\left(\left(x, x^{*}\right)-\left(y, y^{*}\right)\right)$. Thus if $A \subset B$ then $A$ is $q$-positive exactly when $A$ is a nonempty monotone subset of $B$ in the usual sense, and $A$ is maximally $q$-positive exactly when $A$ is a maximally monotone subset of $B$ in the usual sense. We point out that any finite dimensional SSD space of the form described here must have even dimension. Thus cases of Example 3.2 with finite odd dimension cannot be of this form.

Example $3.4\left(\mathbb{R}^{3},\lfloor\cdot, \cdot\rfloor\right)$ is not a SSD space with

$$
\left\lfloor\left(b_{1}, b_{2}, b_{3}\right),\left(c_{1}, c_{2}, c_{3}\right)\right\rfloor:=b_{1} c_{2}+b_{2} c_{3}+b_{3} c_{1} .
$$

(The bilinear form $\lfloor\cdot, \cdot\rfloor$ is not symmetric.)

Definition 3.5 Let $(B,\lfloor\cdot, \cdot\rfloor)$ be a SSD space. If $f \in \mathcal{P C}(B)$ and $f \geq q$ on $B$, we write

$$
\mathcal{P}_{q}(f):=\{b \in B: f(b)=q(b)\} .
$$

We then note from (5) that

$$
\text { if } A \text { is maximally } q \text {-positive then } A=\mathcal{P}_{q}\left(\Phi_{A}\right) \text {. }
$$

If $g \in \mathcal{P C}(B)$ and $g \geq-q$ on $B$ then we write $\mathcal{N}_{q}(g):=\{b \in B: g(b)=-q(b)\}$.

We now introduce the concept of intrinsic conjugate for a SSD space. 
Definition 3.6 If $(B,\lfloor\cdot, \cdot\rfloor)$ is a SSD space and $f \in \mathcal{P C}(B)$, we write $f^{@}$ for the Fenchel conjugate of $f$ with respect to the pairing $\lfloor\cdot, \cdot\rfloor$, that is to say,

$$
\text { for all } c \in B, \quad f^{@}(c):=\sup _{B}[\lfloor\cdot c\rfloor-f] .
$$

The concepts introduced in Definitions 3.5 and 3.6 are related in our next result, which uses a disguised differentiability argument.

Lemma 3.7 Let $(B,\lfloor\cdot, \cdot\rfloor)$ be a SSD space, $f \in \mathcal{P C}(B)$ and $f \geq q$ on B. Then $f^{@}=$ $q$ on $\mathcal{P}_{q}(f)$.

Proof Let $a \in \mathcal{P}_{q}(f)$. Let $\left.\lambda \in\right] 0,1[$. For simplicity in writing, let $\mu:=1-\lambda \in] 0,1[$. Then, for all $b \in B$,

$$
\begin{aligned}
\lambda^{2} q(b)+\lambda \mu\lfloor b, a\rfloor+\mu^{2} q(a) & =q(\lambda b+\mu a) \leq f(\lambda b+\mu a) \\
& \leq \lambda f(b)+\mu f(a)=\lambda f(b)+\mu q(a) .
\end{aligned}
$$

Thus $\lambda^{2} q(b)+\lambda \mu\lfloor b, a\rfloor \leq \lambda f(b)+\lambda \mu q(a)$. Dividing by $\lambda$ and letting $\lambda \rightarrow 0$, we have $\lfloor b, a\rfloor \leq f(b)+q(a)$, that is to say $\lfloor a, b\rfloor-f(b) \leq q(a)$, and, taking the supremum over $b, f^{@}(a) \leq q(a)$. On the other hand, $f^{@}(a) \geq\lfloor a, a\rfloor-f(a)=2 q(a)-q(a)=q(a)$, completing the proof of Lemma 3.7.

The next result gives a basic property of $\Phi_{A}{ }^{\circledR}$.

Lemma 3.8 Let $(B,\lfloor\cdot, \cdot\rfloor)$ be a SSD space and $A$ be a nonempty q-positive subset of $B$. Then $\Phi_{A}{ }^{@} \geq \Phi_{A}$ on $B$.

Proof Let $c \in B$. Then, from (4),

$$
\Phi_{A}(c)=\sup _{B}\left[\lfloor c, \cdot\rfloor-\Phi_{A}\right] \geq \sup _{A}\left[\lfloor c, \cdot\rfloor-\Phi_{A}\right]=\sup _{A}[\lfloor c, \cdot\rfloor-q]=\Phi_{A}(c) .
$$

We now introduce the important concepts of "BC-function" and "TBC-function".

Definition 3.9 Let $(B,\lfloor\cdot, \cdot\rfloor)$ be a SSD space and $f, g \in \mathcal{P C}(B)$. We say that $f$ is a $B C$-function if

$$
b \in B \Longrightarrow f^{@}(b) \geq f(b) \geq q(b) .
$$

"BC" stands for "bigger conjugate". We say that $g$ is a TBC-function if

$$
b \in B \Longrightarrow g^{@}(-b) \geq g(b) \geq-q(b) .
$$

"TBC" stands for "twisted bigger conjugate". Of course, $g$ is a TBC-function if, and only if, $g$ is a BC-function with respect to the $\operatorname{SSD}$ space $(B,-\lfloor\cdot, \cdot\rfloor)$

Lemma 3.10 Let $(B,\lfloor\cdot, \cdot\rfloor)$ be a SSD space and $f \in \mathcal{P C}(B)$ be a BC-function. Then

$$
\mathcal{P}_{q}\left(f^{@}\right)=\mathcal{P}_{q}(f) .
$$

Proof This follows from Lemma 3.7 and the inclusion $\mathcal{P}_{q}\left(f^{@}\right) \subset \mathcal{P}_{q}(f)$, which is immediate from (7).

Theorem 3.11 Let $(B,\lfloor\cdot, \cdot\rfloor)$ be a SSD space and $A$ be a maximally $q$-positive subset of $B$. Then $\Phi_{A}$ is a BC-function and $\mathcal{P}_{q}\left(\Phi_{A}{ }^{@}\right)=\mathcal{P}_{q}\left(\Phi_{A}\right)=A$. 
Proof The first assertion follows from Lemma 3.8 and (5), and the second assertion is immediate from Lemma 3.10 and (6).

Remark 3.12 We shall see by combining (9) and Remark 5.5 that there may exist a function that is both a BC-function and a TBC-function but not of the form $\Phi_{A}$ for any nonempty $q$-positive set $A$.

We now give two computational results. In the first of these, we investigate the "translation" of a BC-function. This result will be used in the "pos-neg" theorems, Theorems 4.3 and 4.6.

Lemma 3.13 Let $(B,\lfloor\cdot, \cdot\rfloor)$ be a SSD space, $f \in \mathcal{P C}(B)$ be a BC-function and $c \in B$. We define $f_{c} \in \mathcal{P C}(B)$ by $f_{c}:=f(\cdot+c)-\lfloor\cdot, c\rfloor-q(c)$. Then $f_{c}$ is a BC-function, $\operatorname{dom} f_{c}=\operatorname{dom} f-c$ and $\mathcal{P}_{q}\left(f_{c}\right)=\mathcal{P}_{q}(f)-c$.

Proof For all $b \in B$,

$$
\begin{aligned}
f_{c}^{@}(b) & =\sup _{d \in B}[\lfloor d, b\rfloor+\lfloor d, c\rfloor+q(c)-f(d+c)] \\
& =\sup _{e \in B}[\lfloor e-c, b+c\rfloor+q(c)-f(e)] \\
& =\sup _{e \in B}[\lfloor e, b+c\rfloor-\lfloor c, b\rfloor-f(e)]-q(c) \\
& =f^{@}(b+c)-\lfloor c, b\rfloor-q(c) .
\end{aligned}
$$

It follows from (7) that $f_{c}{ }^{@}(b) \geq f(b+c)-\lfloor b, c\rfloor-q(c)=f_{c}(b)$ and

$$
f_{c}(b)=f(b+c)-\lfloor b, c\rfloor-q(c) \geq q(b+c)-\lfloor b, c\rfloor-q(c)=q(b) .
$$

Consequently, $f_{c}$ is a BC-function. It is obvious that $\operatorname{dom} f_{c}=\operatorname{dom} f-c$. Further, since

$$
\begin{aligned}
b \in \mathcal{P}_{q}\left(f_{c}\right) & \Longleftrightarrow f(b+c)-\lfloor b, c\rfloor-q(c)=q(b) \\
& \Longleftrightarrow f(b+c)=q(b+c) \Longleftrightarrow b+c \in \mathcal{P}_{q}(f),
\end{aligned}
$$

we have $\mathcal{P}_{q}\left(f_{c}\right)=\mathcal{P}_{q}(f)-c$, as required.

In the second computational result, we show how we can always obtain a TBC-function from a BC-function by an appropriate linear transformation.

Lemma 3.14 Let $(B,\lfloor\cdot, \cdot\rfloor)$ be a SSD space, $\rho: B \rightarrow B$ be a linear surjection such that, for all $b, c \in B,\lfloor\rho(b), \rho(c)\rfloor=\lfloor b,-c\rfloor$, and $g \in \mathcal{P C}(B)$ be a BC-function. Then $g \circ \rho$ and $g \circ(-\rho)$ are TBC-functions, $\rho \operatorname{dom}(g \circ \rho)=\operatorname{dom} g, \rho \mathcal{N}_{q}(g \circ \rho)=\mathcal{P}_{q}(g)$, $-\rho \operatorname{dom}(g \circ(-\rho))=\operatorname{dom} g$ and $-\rho \mathcal{N}_{q}(g \circ(-\rho))=\mathcal{P}_{q}(g)$.

Proof We give the proof for $g \circ \rho$. The proof for $g \circ(-\rho)$ is similar. For all $c \in B$,

$$
\begin{aligned}
(g \circ \rho){ }^{@}(-c) & =\sup _{b \in B}[\lfloor b,-c\rfloor-g(\rho(b))]=\sup _{b \in B}[\lfloor\rho(b), \rho(c)\rfloor-g(\rho(b))] \\
& =\sup _{d \in B}[\lfloor d, \rho(c)\rfloor-g(d)]=g^{@}(\rho(c)) \geq g(\rho(c)) \\
& \geq q(\rho(c))=\frac{1}{2}\lfloor\rho(c), \rho(c)\rfloor=\frac{1}{2}\lfloor c,-c\rfloor=-q(c) .
\end{aligned}
$$

Thus $g \circ \rho$ is a TBC- function. Further,

$$
\begin{aligned}
b \in \rho \operatorname{dom}(g \circ \rho) & \Longleftrightarrow \text { there exists } c \in B \text { such that } g(\rho(c)) \in \mathbb{R} \text { and } b=\rho(c) \\
& \Longleftrightarrow \text { there exists } d \in B \text { such that } g(d) \in \mathbb{R} \text { and } b=d \\
& \Longleftrightarrow g(b) \in \mathbb{R} \Longleftrightarrow b \in \operatorname{dom} g,
\end{aligned}
$$


from which $\rho \operatorname{dom}(g \circ \rho)=\operatorname{dom} g$, and

$$
\begin{aligned}
b \in \rho \mathcal{N}_{q}(g \circ \rho) & \Longleftrightarrow \text { there exists } c \in B \text { such that } g(\rho(c))=-q(c) \text { and } b=\rho(c) \\
& \Longleftrightarrow \text { there exists } c \in B \text { such that } g(\rho(c))=q(\rho(c)) \text { and } b=\rho(c) \\
& \Longleftrightarrow \text { there exists } d \in B \text { such that } g(d)=q(d) \text { and } b=d \\
& \Longleftrightarrow b \in \mathcal{P}_{q}(g),
\end{aligned}
$$

from which $\rho \mathcal{N}_{q}(g \circ \rho)=\mathcal{P}_{q}(g)$.

Lemma 3.15 is a subtler property of the bilinear form $q$. In the situation of Example 3.3, Corollary 3.16 gives us Voisei-Zălinescu [16], Proposition 1.

Lemma 3.15 Let $(B,\lfloor\cdot, \cdot\rfloor)$ be a SSD space, $f \in \mathcal{P C}(B), f \geq q$ on $B$ and $b, c \in B$. Then

$$
-q(b-c) \leq[\sqrt{(f-q)(b)}+\sqrt{(f-q)(c)}]^{2} .
$$

Proof See [14], Lemma 2.6.

Corollary 3.16 Let $(B,\lfloor\cdot, \cdot\rfloor)$ be a SSD space, $f \in \mathcal{P C}(B), f \geq q$ on $B$ and $b, c \in B$. Then

$$
-q(b-c) \leq 2(f-q)(b)+2(f-q)(c) .
$$

Proof This is immediate from Lemma 3.15 and the Cauchy-Schwarz inequality.

Lemma 3.17 is suggested by Burachik-Svaiter, [5], Theorem 3.1, pp. 2381-2382 and Penot, [9], Proposition 4(h) $\Longrightarrow$ (a), pp. 860-861. Lemma 3.17 will be used in Theorem 4.4.

Lemma 3.17 Let $(B,\lfloor\cdot, \cdot\rfloor)$ be a SSD space, $f \in \mathcal{P C}(B), f \geq q$ on $B$ and $\mathcal{P}_{q}(f) \neq \emptyset$. Then $\mathcal{P}_{q}(f)$ is a q-positive subset of $B$.

Proof This is immediate from Lemma 3.15, or Corollary 3.16, or by using the fact that $q$ satisfies the parallelogram law: $x, y \in B \Longrightarrow q(x)+q(y)=\frac{1}{2} q(x+y)+\frac{1}{2} q(x-y)$.

The final result of this section can be thought of as a completion of Lemma 3.8. It will not be used in the sequel.

Lemma 3.18 Let $(B,\lfloor\cdot, \cdot\rfloor)$ be a SSD space and $A$ be a nonempty q-positive subset of $B$. Then $\Phi_{A}{ }^{@} \leq q$ on $A, \Phi_{A}{ }^{@} \geq q$ on $B$, and $\Phi_{A} @=\Phi_{A}$ on $B$.

Proof See [14], Lemma 2.11.

\section{SSDB spaces}

We now introduce the SSDB spaces, a subclass of the class of SSD spaces. Our treatment of the SSD spaces in Sect. 3 has been essentially nontopological. The additional norm structure of the SSDB spaces is essentially what makes maximally monotone multifunctions on a reflexive Banach space much more tractable than those on a general Banach space. We will return to this issue when we consider Example 3.3 in Sect. 5. 
Definition 4.1 We will say that $(B, L \cdot, \cdot,\|\cdot\|)$ is a symmetrically self-dual Banach space $(\operatorname{SSDB}$ space $)$ if $(B,\lfloor\cdot, \cdot\rfloor)$ is a SSD space, $(B,\|\cdot\|)$ is a Banach space, and there exists a linear isometry $\iota$ from $B$ onto $B^{*}$ such that, for all $b, c \in B,\langle b, \iota(c)\rangle=\lfloor b, c\rfloor$. We note then that, for all $f \in \mathcal{P C}(B)$ and $c \in B, f^{@}(c):=\sup _{B}[\lfloor\cdot, c\rfloor-f]=\sup _{B}[\langle\cdot, \iota(c)\rangle-f]=f^{*}(\iota(c))$, that is to say $f^{@}=f^{*} \circ \iota$. It is easy to see that the quadratic form $q$ is continuous and, for all $b \in B,|q(b)|=\frac{1}{2}|\lfloor b, b\rfloor| \leq \frac{1}{2}\|b\|^{2}$.

Let $g_{0}:=\frac{1}{2}\|\cdot\|^{2}$ on $B$. Then, for all $b \in B$,

$$
g_{0}{ }^{@}(b)=g_{0}{ }^{*}(\iota(b))=\frac{1}{2}\|\iota(b)\|^{2}=\frac{1}{2}\|b\|^{2}=g_{0}(b) .
$$

It follows that

$g_{0}$ is both a BC-function and a TBC-function.

Example 4.2 (a) In Example $3.2(\mathrm{a}),(B,\lfloor\cdot, \cdot,\|\cdot\|)$ is a SSDB space under the Hilbert space norm, $q=g_{0}$ and $\mathcal{N}_{q}\left(g_{0}\right)=\{0\}$. (We recall that the set $\mathcal{N}_{q}\left(g_{0}\right)$ was defined in Definition 3.5.)

(b) In Example $3.2(\mathrm{~b}),(B,\lfloor\cdot, \cdot\rfloor,\|\cdot\|)$ is a SSDB space under the Hilbert space norm, $q=-g_{0}$ and $\mathcal{N}_{q}\left(g_{0}\right)=B$.

(c) In Example $3.2(\mathrm{c}),(B,\lfloor\cdot, \cdot\rfloor,\|\cdot\|)$ is a SSDB space under the Euclidean norm and

$$
\mathcal{N}_{q}\left(g_{0}\right)=\left\{\left(b_{1}, b_{2}, b_{3}\right) \in B: b_{1}+b_{2}=0, b_{3}=0\right\} .
$$

Our next result is the "pos-neg theorem of Rockafellar type". This will be used indirectly in Theorem 5.1, which will be used in turn indirectly in our proof of Rockafellar's surjectivity theorem, Theorem 6.3, and the sum theorem, Theorem 6.5. This result first appeared in Simons, [13], Theorem 19.16, pp. 83.

Theorem 4.3 Let $(B,\lfloor\cdot, \cdot\rfloor,\|\cdot\|)$ be a SSDB space, $f \in \mathcal{P C}(B)$ be a BC-function and $g: B \rightarrow \mathbb{R}$ be a continuous TBC-function. Then $\mathcal{P}_{q}(f)-\mathcal{N}_{q}(g)=B$.

Proof Let $c$ be an arbitrary element of $B$. From Lemma 3.13, $f_{c}$ is a BC-function and so, using (7) and (8),

$$
b \in B \Longrightarrow f_{c}(b)+g(b) \geq q(b)-q(b)=0 .
$$

Rockafellar's version of the Fenchel duality theorem, Theorem 2.1, and the surjectivity of $\iota$ now give $b \in B$ such that $f_{c}{ }^{*}(\iota(b))+g^{*}(-\iota(b)) \leq 0$, that is to say $f_{c}{ }^{@}(b)+g^{@}(-b) \leq 0$. From (7) and (8) again, $f_{c}(b)+g(b) \leq 0=q(b)-q(b)$. From (7) and (8) for a third time, $f_{c}(b)=q(b)$ and $g(b)=-q(b)$, that is to say, using Lemma 3.13, $b \in \mathcal{P}_{q}\left(f_{c}\right)=\mathcal{P}_{q}(f)-c$ and also $b \in \mathcal{N}_{q}(g)$. But then $c=(c+b)-b \in \mathcal{P}_{q}(f)-\mathcal{N}_{q}(g)$. This completes the proof of Theorem 4.3.

Theorem 4.4 Let $(B,\lfloor\cdot, \cdot\rfloor,\|\cdot\|)$ be a SSDB space. Then:

(a) Let $f \in \mathcal{P C}(B)$ be a BC-function. Then $\mathcal{P}_{q}(f)-\mathcal{N}_{q}\left(g_{0}\right)=B$.

(b) Let $A$ be a q-positive subset of $B$ and $A-\mathcal{N}_{q}\left(g_{0}\right)=B$. Then $A$ is maximally q-positive.

(c) Let $f \in \mathcal{P C}(B)$ be a BC-function. Then $\mathcal{P}_{q}(f)$ is maximally $q$-positive. Furthermore, $\mathcal{P}_{q}\left(f^{@}\right)=\mathcal{P}_{q}(f)$.

(d) Let $A$ be a q-positive subset of $B$. Then $A$ is maximally q-positive if, and only if $A-\mathcal{N}_{q}\left(g_{0}\right)=B$. 
Proof (a) This follows from (9) and Theorem 4.3.

(b) Suppose that $b \in B$ and $A \cup\{b\}$ is $q$-positive. By hypothesis, there exists $a \in A$ such that $a-b \in \mathcal{N}_{q}\left(g_{0}\right)$. Thus $\frac{1}{2}\|a-b\|^{2}=-q(a-b)$. Since $A \cup\{b\}$ is $q$-positive, $q(a-b) \geq 0$, and so $\frac{1}{2}\|a-b\|^{2} \leq 0$. Thus $b=a \in A$.

(c) From (a) and Lemma 3.17, $\mathcal{P}_{q}(f)$ is nonempty and $q$-positive. (c) now follows from (a), (b) and Lemma 3.10.

(d) We have already established ( $\Longleftarrow$ ) in (b). Suppose, conversely, that $A$ is maximally $q$-positive. It follows from Theorem 3.11 that $\Phi_{A}$ is a BC-function and $\mathcal{P}_{q}\left(\Phi_{A}\right)=A$. Thus, from (a), $A-\mathcal{N}_{q}\left(g_{0}\right)=\mathcal{P}_{q}\left(\Phi_{A}\right)-\mathcal{N}_{q}\left(g_{0}\right)=B$.

Remark 4.5 We note from (9) and Theorem 4.4(c) that $\mathcal{P}_{q}\left(g_{0}\right)$ is maximally $q$-positive and $\mathcal{P}_{q}\left(g_{0}{ }^{\circledR}\right)=\mathcal{P}_{q}\left(g_{0}\right)$. Now suppose that $a \in \mathcal{P}_{q}\left(g_{0}\right)$ and $b \in B$. From the Fenchel-Young inequality, $\lfloor a, b\rfloor-q(a)=\lfloor a, b\rfloor-g_{0}{ }^{@}(a) \leq g_{0}(b)$. Taking the supremum over $a \in \mathcal{P}_{q}\left(g_{0}\right)$, we deduce that $\Phi_{\mathcal{P}_{q}\left(g_{0}\right)} \leq g_{0}$ on $B$. In particular, dom $\Phi_{\mathcal{P}_{q}\left(g_{0}\right)}=B$.

The next result in this section is the "pos-neg theorem of Attouch-Brezis type". A stronger result was proved in Simons, [13], Theorem 21.12, pp. 93.

Theorem 4.6 Let $(B,\lfloor\cdot, \cdot\rfloor,\|\cdot\|)$ be a SSDB space, $f \in \mathcal{P C} \mathcal{L S C}(B)$ be a BC-function, and $g \in \mathcal{P C} \mathcal{L S C}(B)$ be a TBC-function. Then $\operatorname{dom} f-\operatorname{dom} g=B \Longleftrightarrow \mathcal{P}_{q}(f)-\mathcal{N}_{q}(g)=B$.

Proof Since the implication ( $\Longleftarrow$ ) is trivial, we only have to prove $(\Longrightarrow)$. Let $c$ be an arbitrary element of $B$. Then, from Lemma 3.13,

$$
\operatorname{dom} f_{c}-\operatorname{dom} g=\operatorname{dom} f-c-\operatorname{dom} g=B-c=B,
$$

and so $\bigcup_{\lambda>0} \lambda\left[\operatorname{dom} f_{c}-\operatorname{dom} g\right]=B$. From Lemma 3.13, $f_{c}$ is a BC-function and so, using (7) and (8),

$$
b \in B \Longrightarrow f_{c}(b)+g(b) \geq q(b)-q(b)=0 .
$$

The Attouch-Brezis theorem, Theorem 2.2, and the surjectivity of $\iota$ now give $b \in B$ such that $f_{c}{ }^{*}(\iota(b))+g^{*}(-\iota(b)) \leq 0$, that is to say $f_{c}{ }^{@}(b)+g^{@}(-b) \leq 0$. From $(7)$ and $(8)$ again, $f_{c}(b)+g(b) \leq 0=q(b)-q(b)$. From (7) and (8) for a third time, $f_{c}(b)=q(b)$ and $g(b)=-q(b)$, that is to say, using Lemma 3.13, $b \in \mathcal{P}_{q}\left(f_{c}\right)=\mathcal{P}_{q}(f)-c$ and also $b \in \mathcal{N}_{q}(g)$. But then $c=(c+b)-b \in \mathcal{P}_{q}(f)-\mathcal{N}_{q}(g)$. This completes the proof of Theorem 4.6.

Corollary 4.7 below is the form in which we will actually apply Theorem 4.6 to abstract Hammerstein theorems in Theorem 7.1.

Corollary 4.7 Let $(B,\lfloor\cdot, \cdot\rfloor,\|\cdot\|)$ be a SSDB space, $f, g \in \mathcal{P C} \mathcal{L S C}(B)$ and $f, g$ be $B C$-functions. Suppose, further, that $\rho: B \rightarrow B$ is a continuous linear bijection such that, for all $b, c \in B,\lfloor\rho(b), \rho(c)\rfloor=\lfloor b,-c\rfloor$. Then

$$
\operatorname{dom} f-\rho^{-1} \operatorname{dom} g=B \Longleftrightarrow \mathcal{P}_{q}(f)-\rho^{-1} \mathcal{P}_{q}(g)=B
$$

and

$$
\operatorname{dom} f+\rho^{-1} \operatorname{dom} g=B \Longleftrightarrow \mathcal{P}_{q}(f)+\rho^{-1} \mathcal{P}_{q}(g)=B .
$$

Proof This is immediate from Theorem 4.6 and Lemma 3.14. 


\section{BC-functions on $E \times E^{*}$}

From now on, $E$ is a nonzero reflexive Banach space and $E^{*}$ is its topological dual space. As observed in Example 3.3, $\left(E \times E^{*},\lfloor\cdot, \cdot\rfloor\right)$ is a SSD space with $q\left(x, x^{*}\right)=\left\langle x, x^{*}\right\rangle$, and if $A \subset E \times E^{*}$ then $A$ is $q$-positive exactly when $A$ is a nonempty monotone set in the usual sense, and $A$ is maximally $q$-positive exactly when $A$ is a maximally monotone set of in the usual sense. We norm $E \times E^{*}$ by $\left\|\left(x, x^{*}\right)\right\|:=\sqrt{\|x\|^{2}+\left\|x^{*}\right\|^{2}}$. Then

$$
\left(E \times E^{*},\|\cdot\|\right)^{*}=\left(E^{*} \times E,\|\cdot\|\right),
$$

under the duality $\left\langle\left(x, x^{*}\right),\left(y^{*}, y\right)\right\rangle:=\left\langle x, y^{*}\right\rangle+\left\langle y, x^{*}\right\rangle$. Combining this with the formula in Example 3.3, we have $\left\langle\left(x, x^{*}\right),\left(y^{*}, y\right)\right\rangle=\left\lfloor\left(x, x^{*}\right),\left(y, y^{*}\right)\right\rfloor$, and so $\iota\left(y, y^{*}\right)=\left(y^{*}, y\right)$. It is easily seen from these relationships that $\left(E \times E^{*},\lfloor\cdot, \cdot\rfloor,\|\cdot\|\right)$ is a SSDB space. Further, $\left(x, x^{*}\right) \in \mathcal{P}_{q}\left(g_{0}\right) \Longleftrightarrow \frac{1}{2}\|x\|^{2}+\frac{1}{2}\left\|x^{*}\right\|^{2}=\left\langle x, x^{*}\right\rangle \Longleftrightarrow\left(x, x^{*}\right) \in G(J)$, and $\left(x, x^{*}\right) \in$ $\mathcal{N}_{q}\left(g_{0}\right) \Longleftrightarrow \frac{1}{2}\|x\|^{2}+\frac{1}{2}\left\|x^{*}\right\|^{2}=-\left\langle x, x^{*}\right\rangle \Longleftrightarrow\left(x, x^{*}\right) \in G(-J)$, where $J: E \rightrightarrows E^{*}$ is the duality map. Thus we obtain the following fundamental result:

Theorem 5.1 Let $E$ be a nonzero reflexive Banach space. Then:

(a) If $f \in \mathcal{P C}\left(E \times E^{*}\right)$ and $f$ is a $B C$-function then $\mathcal{P}_{q}(f)$ is maximally monotone, $\mathcal{P}_{q}\left(f^{@}\right)=\mathcal{P}_{q}(f)$ and $\mathcal{P}_{q}(f)-G(-J)=B$.

(b) If $A$ is a monotone subset of $E \times E^{*}$ then $A$ is maximally monotone if, and only if, $A-G(-J)=E \times E^{*}$.

Proof (a) is immediate from Theorem 4.4(a,c), and (b) is immediate from Theorem 4.4(d).

Remark 5.2 If $f \in \mathcal{P C} \mathcal{L S C}(E)$ and, for all $\left(x, x^{*}\right) \in B, h\left(x, x^{*}\right):=f(x)+f^{*}\left(x^{*}\right)$ then the Fenchel-Moreau theorem and the Fenchel-Young inequality imply that $h$ is a BC-function on $E \times E^{*}$. Since $\mathcal{P}_{q}(h)=G(\partial f)$, Theorem 5.1(a) gives us that $\partial f$ is maximally monotone. (Remember that we are assuming that $E$ is reflexive!)

We note that the "partial episum theorem" of Theorem 5.3 below can also be deduced from Penot-Zălinescu, [10], Corollary 3.7.

Theorem 5.3 Let $E$ be a nonzero reflexive Banach space, $f, g \in \mathcal{P C} \mathcal{L} \mathcal{S C}\left(E \times E^{*}\right)$ be $B C$-functions, $\bigcup_{\lambda>0} \lambda\left[\pi_{1} \operatorname{dom} f-\pi_{1} \operatorname{dom} g\right]$ be a closed linear subspace of $E$ and, for all $\left(x, x^{*}\right) \in E \times E^{*}$,

$$
h\left(x, x^{*}\right):=\inf \left\{f\left(x, s^{*}\right)+g\left(x, t^{*}\right): s^{*}, t^{*} \in E^{*}, s^{*}+t^{*}=x^{*}\right\} .
$$

Then $h$ is a BC-function, $\mathcal{P}_{q}\left(h^{@}\right)=\left\{\left(x, s^{*}+t^{*}\right):\left(x, s^{*}\right) \in \mathcal{P}_{q}\left(f^{@}\right),\left(x, t^{*}\right) \in \mathcal{P}_{q}\left(g^{@}\right)\right\}$, and

$$
\mathcal{P}_{q}(h)=\left\{\left(x, s^{*}+t^{*}\right):\left(x, s^{*}\right) \in \mathcal{P}_{q}(f),\left(x, t^{*}\right) \in \mathcal{P}_{q}(g)\right\} .
$$

Proof Since $f \geq q$ and $g \geq q$ on $E \times E^{*}$, (10) implies that, for all $\left(x, x^{*}\right) \in E \times E^{*}$,

$$
h\left(x, x^{*}\right) \geq \inf \left\{\left\langle x, s^{*}\right\rangle+\left\langle x, t^{*}\right\rangle: s^{*}, t^{*} \in E^{*}, s^{*}+t^{*}=x^{*}\right\}=\left\langle x, x^{*}\right\rangle=q\left(x, x^{*}\right),
$$

and then Theorem 2.4 and the fact that $f^{@} \geq f$ and $g^{@} \geq g$ on $E \times E^{*}$ give

$$
\begin{aligned}
h^{@}\left(x, x^{*}\right) & =h^{*}\left(x^{*}, x\right)=\min \left\{f^{*}\left(s^{*}, x\right)+g^{*}\left(t^{*}, x\right): s^{*}, t^{*} \in E^{*}, s^{*}+t^{*}=x^{*}\right\} \\
& =\min \left\{f^{@}\left(x, s^{*}\right)+g^{@}\left(x, t^{*}\right): s^{*}, t^{*} \in E^{*}, s^{*}+t^{*}=x^{*}\right\} \\
& \geq \inf \left\{f\left(x, s^{*}\right)+g\left(x, t^{*}\right): s^{*}, t^{*} \in E^{*}, s^{*}+t^{*}=x^{*}\right\}=h\left(x, x^{*}\right) .
\end{aligned}
$$


Thus $h$ is a BC-function, the required characterization of $\mathcal{P}_{q}\left(h^{@}\right)$ is immediate from (11), and the final assertion now follows from three applications of Theorem 5.1(a).

Theorem 5.4 Let $E$ be a nonzero reflexive Banach space, $f, g \in \mathcal{P C} \mathcal{L S C}\left(E \times E^{*}\right)$ be $B C$-functions and $\bigcup_{\lambda>0} \lambda\left[\pi_{1} \operatorname{dom} f-\pi_{1} \operatorname{dom} g\right]$ be a closed linear subspace of $E$. Then

$$
\left\{\left(x, s^{*}+t^{*}\right):\left(x, s^{*}\right) \in \mathcal{P}_{q}(f),\left(x, t^{*}\right) \in \mathcal{P}_{q}(g)\right\}
$$

is a maximally monotone subset of $E \times E^{*}$.

Proof This is immediate from Theorems 5.1(a) and 5.3.

Remark 5.5 The question arises naturally as to whether there can exist a $q$-positive subset $A$ of $B$ such that $\Phi_{A}=g_{0}$. It would then follow from (4) that $g_{0}=q$ on A, and so $A \subset \mathcal{P}_{q}\left(g_{0}\right)$. Thus, for all $b \in B$, (2) would give

$$
g_{0}(b)=\Phi_{A}(b)=\sup _{A}[\lfloor\cdot, b\rfloor-q]=\sup _{A}\left[\lfloor\cdot, b\rfloor-g_{0}\right] \leq \sup _{\mathcal{P}_{q}\left(g_{0}\right)}\left[\lfloor\cdot, b\rfloor-g_{0}\right] .
$$

Now let $H$ be a nonzero Hilbert space, and consider the SSDB space $(H \times H,\lfloor\cdot, \cdot\rfloor,\|\cdot\|)$, as described at the beginning of this section. Then $\left(x, x^{*}\right) \in \mathcal{P}_{q}\left(g_{0}\right) \Longleftrightarrow x^{*}=x$. Let $y \in H \backslash\{0\}$. Then (12) would give

$$
\|y\|^{2}=g_{0}(y,-y) \leq \sup _{x \in H}\left[\lfloor(x, x),(y,-y)\rfloor-g_{0}(x, x)\right]=\sup _{x \in H}\left[-g_{0}(x, x)\right] \leq 0 .
$$

Since this is manifestly impossible, there cannot exist a $q$-positive subset $A$ of $B$ such that $\Phi_{A}=g_{0}$. This example is an extension of [13], Example 19.20, p. 85.

\section{Monotone multifunctions on reflexive banach spaces}

Let $E$ be a nonzero reflexive Banach space. If $S: E \rightrightarrows E^{*}$ is a multifunction then we use the standard notation $G(S):=\left\{\left(x, x^{*}\right) \in E \times E^{*}: x^{*} \in S x\right\}$. We also write $D(S):=$ $\{x \in E: S x \neq \emptyset\}=\pi_{1} G(S)$. If $S$ is monotone and $G(S)$ is nonempty, we define the Fitzpatrick functions, $\varphi_{S}$, associated with $S$ by

$$
\varphi_{S}\left(x, x^{*}\right):=\Phi_{G(S)}\left(x, x^{*}\right)=\sup _{\left(s, s^{*}\right) \in G(S)}\left[\left\langle x, s^{*}\right\rangle+\left\langle s, x^{*}\right\rangle-\left\langle s, s^{*}\right\rangle\right] .
$$

The function $\varphi_{A}$ was introduced by Fitzpatrick in [6], Definition 3.1, pp. 61. The following result was first proved in [6], Corollary 3.9, pp. 62 and [6], Proposition 4.2, pp. 63-64.

Theorem 6.1 E be a nonzero reflexive Banach space and $S: E \rightrightarrows E^{*}$ be maximally monotone. Then $\varphi_{S}$ is a BC-function and $\mathcal{P}_{q}\left(\varphi_{S}{ }^{\circledR}\right)=\mathcal{P}_{q}\left(\varphi_{S}\right)=G(S)$.

Proof This is immediate from Theorem 3.11.

The following result will be useful in Corollary 6.6.

Lemma 6.2 E be a nonzero reflexive Banach space and $S: E \rightrightarrows E^{*}$ be maximally monotone. Then $D(S) \subset \pi_{1} \operatorname{dom} \varphi_{S}$.

Proof From Theorem 6.1 and the finite-valuedness of $q, G(S)=\mathcal{P}_{q}\left(\varphi_{S}\right) \subset \operatorname{dom} \varphi_{S}$, from which $D(S)=\pi_{1} G(S) \subset \pi_{1} \operatorname{dom} \varphi_{S}$. 
If $S, T: E \rightrightarrows F$ then, for all $x \in E$, we use the following standard notation:

$$
(S+T) x:=\{y+z: y \in S x, z \in T x\} .
$$

Theorem 6.3 is "Rockafellar's surjectivity theorem"—-see [12], Proposition 1, p. 77 for the original proof depending ultimately on Brouwer's fixed-point theorem and an Asplund renorming. The proof given here is a simplification of that given in Simons-Zălinescu, [15], Theorem 3.1(b), p. 8, and appeared in Simons, [13], Theorem 29.6, p. 119. These references also provide a number of formulae for the exact value of $\min \{\|x\|: x \in E,(S+J) x \ni 0\}$ in terms of $\varphi_{G(S)}$. Here is one of them:

$$
\min \{\|x\|: x \in E,(S+J) x \ni 0\}=\frac{1}{\sqrt{2}} \sup _{b \in E \times E^{*}}\left[\|b\|-\sqrt{2 \varphi_{S}(b)+\|b\|^{2}}\right] \vee 0 .
$$

Theorem 6.3 Let $E$ be a nonzero reflexive Banach space and $S: E \rightrightarrows E^{*}$ be maximally monotone. Then $(S+J)(E)=E^{*}$.

Proof Let $y^{*}$ be an arbitrary element of $E^{*}$. From Theorem 5.1(b) (with $A:=G(S)$ ), $\left(0, y^{*}\right) \in G(S)-G(-J)$. Thus there exist $\left(s, s^{*}\right) \in G(S)$ and $\left(x, x^{*}\right) \in G(J)$ such that $\left(0, y^{*}\right)=\left(s, s^{*}\right)-\left(x,-x^{*}\right)$. But then $x=s$, and so

$$
y^{*}=s^{*}+x^{*} \in S x+J x=(S+J) x \subset(S+J)(E) .
$$

Remark 6.4 We note that if $J$ and $J^{-1}$ are single-valued then the converse of Theorem 6.3 holds, that is to say $(S+J)(E)=E^{*} \Longrightarrow S$ is maximally monotone, however this fails in general. See Simons, [13], Remark 29.7, pp. 120-121. It is interesting to observe that, even when the converse of Theorem 6.3 fails, the necessary and sufficient condition for maximal monotonicity given in Theorem 5.1(b) remains true.

We now come to the "sum theorem". More general results can be found in Bot-CsetnekWanka, [3] and Boţ-Grad-Wanka, [4].

Theorem 6.5 Let $E$ be a nonzero reflexive Banach space, $S, T: E \rightrightarrows E^{*}$ be maximally monotone and $\bigcup_{\lambda>0} \lambda\left[\pi_{1} \operatorname{dom} \varphi_{S}-\pi_{1} \operatorname{dom} \varphi_{T}\right]$ be a closed linear subspace of $E$. Then $S+T$ is maximally monotone.

Proof From Theorem 6.1, $\varphi_{S}$ and $\varphi_{T}$ are BC-functions, $\mathcal{P}_{q}\left(\varphi_{S}\right)=G(S)$ and $\mathcal{P}_{q}\left(\varphi_{T}\right)=$ $G(T)$. Theorem 5.4 with $f:=\varphi_{S}$ and $g:=\varphi_{T}$ now implies that

$$
\left\{\left(x, s^{*}+t^{*}\right):\left(x, s^{*}\right) \in G(S),\left(x, t^{*}\right) \in G(T)\right\}=G(S+T)
$$

is a maximally monotone subset of $E \times E^{*}$. This completes the proof of Theorem 6.5.

The following consequence of Theorem 6.5 is useful in applications:

Corollary 6.6 Let $E$ be a nonzero reflexive Banach space, $S, T: E \rightrightarrows E^{*}$ be maximally monotone and $\bigcup_{\lambda>0} \lambda[D(S)-D(T)]=E$. Then $S+T$ is maximally monotone.

Proof This is immediate from Lemma 6.2 and Theorem 6.5.

Our next result is “Rockafellar's sum theorem”. It follows immediately from Corollary 6.6. 
Corollary 6.7 Let $E$ be a nonzero reflexive Banach space, $S, T: E \rightrightarrows E^{*}$ be maximally monotone and $D(S) \cap$ int $D(T) \neq \emptyset$. Then $S+T$ is maximally monotone.

A number of other sufficient conditions have been given for the sum of maximally monotone multifunctions on a reflexive Banach space to be maximally monotone. Many of them are contained in the following "Sandwiched closed subspace theorem", which first appeared in Simons-Zălinescu, [15], Theorem 5.5, pp. 13.

Theorem 6.8 Let $E$ be a nonzero reflexive Banach space, $S, T: E \rightrightarrows E^{*}$ be maximally monotone, and suppose that there exists a closed linear subspace $F$ of $E$ such that

$$
D(S)-D(T) \subset F \subset \bigcup_{\lambda>0} \lambda\left[\pi_{1} \operatorname{dom} \varphi_{S}-\pi_{1} \operatorname{dom} \varphi_{T}\right] .
$$

Then $S+T$ is maximally monotone. Furthermore, for all $\varepsilon>0$,

$$
D(S)-D(T) \subset \pi_{1} \operatorname{dom} \varphi_{S}-\pi_{1} \operatorname{dom} \varphi_{T} \subset(1+\varepsilon)(D(S)-D(T)),
$$

(that is to say, the sets $\pi_{1} \operatorname{dom} \varphi_{S}-\pi_{1} \operatorname{dom} \varphi_{T}$ and $D(S)-D(T)$ are almost identical) and

$$
\bigcup_{\lambda>0} \lambda\left[\pi_{1} \operatorname{dom} \varphi_{S}-\pi_{1} \operatorname{dom} \varphi_{T}\right]=\bigcup_{\lambda>0} \lambda[D(S)-D(T)] .
$$

\section{An abstract hammerstein theorem}

Let $E$ be a nonzero reflexive Banach space. We define the reflection maps $\rho_{1}, \rho_{2}$ on $E \times E^{*}$ by $\rho_{1}\left(x, x^{*}\right):=\left(-x, x^{*}\right)$ and $\rho_{2}\left(x, x^{*}\right):=\left(x,-x^{*}\right)$. Our first result is the " $\rho_{1}$-transversality theorem".

Theorem 7.1 Let $E$ be a nonzero reflexive Banach space, $f, g \in \mathcal{P C} \mathcal{L S C}\left(E \times E^{*}\right)$ and $f, g$ be BC-functions. Then:

$$
\operatorname{dom} f+\rho_{1} \operatorname{dom} g=E \times E^{*} \Longleftrightarrow \mathcal{P}_{q}(f)+\rho_{1} \mathcal{P}_{q}(g)=E \times E^{*}
$$

and

$$
\operatorname{dom} f-\rho_{1} \operatorname{dom} g=E \times E^{*} \Longleftrightarrow \mathcal{P}_{q}(f)-\rho_{1} \mathcal{P}_{q}(g)=E \times E^{*} .
$$

Proof This follows from Corollary 4.7 with $\rho:=\rho_{2}$, and the fact that $\rho_{1}=-\rho_{2}^{-1}$.

We give the next two results for completeness, though more general results are known. See, for instance, Zălinescu [18], Theorem 3 and Corollary 4.

Theorem 7.2 Let $E$ be a nonzero reflexive Banach space, $S, T: E \rightrightarrows E^{*}$ be maximally monotone and $\operatorname{dom} \varphi_{S}+\rho_{1} \operatorname{dom} \varphi_{T}=E \times E^{*}$. Then $(S+T)(E)=E^{*}$.

Proof Let $y^{*}$ be an arbitrary element of $E^{*}$. From Theorem 7.1 (with $f:=\varphi_{S}$ and $g:=\varphi_{T}$ ) and Theorem 6.1, $\left(0, y^{*}\right) \in G(S)+\rho_{1} G(T)$. Thus there exist $\left(s, s^{*}\right) \in G(S)$ and $\left(x, x^{*}\right) \in$ $G(T)$ such that $\left(0, y^{*}\right)=\left(s, s^{*}\right)+\left(-x, x^{*}\right)$. But then $x=s$, and so

$$
y^{*}=s^{*}+x^{*} \in S x+T x=(S+T) x \subset(S+T)(E) .
$$

Since $G(J)=\mathcal{P}_{q}\left(g_{0}\right)$, it follows that $\varphi_{J}=\Phi_{G(J)}=\Phi_{\mathcal{P}_{q}\left(g_{0}\right)}$. Thus Remark 4.5 implies that $\operatorname{dom} \varphi_{J}=E \times E^{*}$. Consequently, the following result generalizes Theorem 6.3. Compare with [7], Theorem 2 ((1) $\Longrightarrow(2))$. 
Corollary 7.3 Let $E$ be a nonzero reflexive Banach space, $S, T: E \rightrightarrows E^{*}$ be maximally monotone and $\operatorname{dom} \varphi_{T}=E \times E^{*}$. Then $(S+T)(E)=E^{*}$.

Proof This is immediate from Theorem 7.2.

Theorem 7.4 Let $E$ be a nonzero reflexive Banach space, $f, g \in \mathcal{P C} \mathcal{L S C}\left(E \times E^{*}\right)$ be $B C$-functions, and $w^{*} \in E^{*}$ be such that $E \times\left\{w^{*}\right\} \subset \operatorname{dom} f$ and $\pi_{2} \operatorname{dom} g=E^{*}$. Then:

(a) $\mathcal{P}_{q}(f)+\rho_{1} \mathcal{P}_{q}(g)=E \times E^{*}$ and $\mathcal{P}_{q}(f)-\rho_{1} \mathcal{P}_{q}(g)=E \times E^{*}$.

(b) If $x \in E$ then there exist $\left(y, y^{*}\right) \in \mathcal{P}_{q}(f)$ and $\left(z, y^{*}\right) \in \mathcal{P}_{q}(g)$ such that $y+z=x$.

(c) If $x^{*} \in E^{*}$ then there exist $\left(y, y^{*}\right) \in \mathcal{P}_{q}(f)$ and $\left(y, z^{*}\right) \in \mathcal{P}_{q}(g)$ such that $y^{*}+z^{*}=$ $x^{*}$.

Proof (a) Let $\left(x, x^{*}\right)$ be an arbitrary element of $E \times E^{*}$. Since $\pi_{2} \operatorname{dom} g=E^{*}$, there exist $y, z \in E$ such that $\left(y, x^{*}-w^{*}\right) \in \operatorname{dom} g$ and $\left(z, w^{*}-x^{*}\right) \in \operatorname{dom} g$. But $\left(x+y, w^{*}\right) \in \operatorname{dom} f$ and $\left(x-z, w^{*}\right) \in \operatorname{dom} f$, hence

$$
\left(x, x^{*}\right)=\left(x+y, w^{*}\right)+\rho_{1}\left(y, x^{*}-w^{*}\right) \in \operatorname{dom} f+\rho_{1} \operatorname{dom} g
$$

and

$$
\left(x, x^{*}\right)=\left(x-z, w^{*}\right)-\rho_{1}\left(z, w^{*}-x^{*}\right) \in \operatorname{dom} f-\rho_{1} \operatorname{dom} g .
$$

Thus we have proved that $\operatorname{dom} f+\rho_{1} \operatorname{dom} g=E \times E^{*}$ and $\operatorname{dom} f-\rho_{1} \operatorname{dom} g=$ $E \times E^{*}$, and (a) follows from Theorem 7.1.

(b) It follows from (a) that there exist $\left(y, y^{*}\right) \in \mathcal{P}_{q}(f)$ and $\left(z, z^{*}\right) \in \mathcal{P}_{q}(g)$ such that $\left(y, y^{*}\right)+\left(z,-z^{*}\right)=(x, 0)$. But then $z^{*}=y^{*}$ and $y+z=x$.

(c) It follows from (a) that there exist $\left(y, y^{*}\right) \in \mathcal{P}_{q}(f)$ and $\left(z, z^{*}\right) \in \mathcal{P}_{q}(g)$ such that $\left(y, y^{*}\right)-\left(z,-z^{*}\right)=\left(0, x^{*}\right)$. But then $z=y$ and $y^{*}+z^{*}=x^{*}$.

We now reverse the direction of $T$.

Theorem 7.5 Let $E$ be a nonzero reflexive Banach space and $S: E \rightrightarrows E^{*}$ and $T: E^{*} \rightrightarrows E$ be maximally monotone. Suppose that $\pi_{1} \operatorname{dom} \varphi_{T}=E^{*}$ and there exists $w^{*} \in E^{*}$ such that $E \times\left\{w^{*}\right\} \subset \operatorname{dom} \varphi_{S}$. Then:

(a) If $I_{E}$ is the identity map on $E,\left(I_{E}+T S\right)(E)=E$.

(b) If $I_{E^{*}}$ is the identity map on $E^{*},\left(I_{E^{*}}+S T\right)\left(E^{*}\right)=E^{*}$.

Proof Let $f:=\varphi_{S}$ and $g:=\varphi_{T^{-1}}$, so that $\pi_{2} \operatorname{dom} g=E^{*}$.

(a) Let $x$ be an arbitrary element of $E$. From Theorems 7.4(b) and 6.1, there exist $\left(y, y^{*}\right) \in$ $G(S)$ and $\left(y^{*}, z\right) \in G(T)$ such that $y+z=x$. Thus $z \in T y^{*} \subset T S y$ and $x=y+z \in$ $\left(I_{E}+T S\right)(y) \subset\left(I_{E}+T S\right)(E)$.

(b) Let $x^{*}$ be an arbitrary element of $E^{*}$. From Theorems 7.4(c) and 6.1, there exist $\left(y, y^{*}\right) \in G(S)$ and $\left(z^{*}, y\right) \in G(T)$ such that $y^{*}+z^{*}=x^{*}$. Thus $y^{*} \in S y \subset S T z^{*}$ and $x^{*}=z^{*}+y^{*} \in\left(I_{E^{*}}+S T\right)\left(z^{*}\right) \subset\left(I_{E^{*}}+S T\right)\left(E^{*}\right)$.

The next result is a considerable generalization of [19], Theorem 32.O, p. 909 which, in turn, was applied to Hammerstein integral equations. See [13], Remark 30.5, p. 124 for a more complete discussion. 
Theorem 7.6 Let $E$ be a nonzero reflexive Banach space and $S: E \rightrightarrows E^{*}$ and $T: E^{*} \rightrightarrows E$ be maximal monotone. Suppose that either $\pi_{1} \operatorname{dom} \varphi_{T}=E^{*}$ and there exists $w^{*} \in E^{*}$ such that $E \times\left\{w^{*}\right\} \subset \operatorname{dom} \varphi_{S}$ or $\pi_{1} \operatorname{dom} \varphi_{S}=E$ and there exists $w \in E$ such that $E \times\{w\} \subset \operatorname{dom} \varphi_{T}$. Then $\left(I_{E}+T S\right)(E)=E$.

Proof The first case has already been established in Theorem 7.5(a), while the second case follows from Theorem 7.5(b), with $E$ replaced by $E^{*}$, and the roles of $S$ and $T$ interchanged.

Remark 7.7 In the recent paper [2], Jonathan Borwein has written a survey of the history of monotonicity (even in the nonreflexive case) over the past 50 years.

Open Access This article is distributed under the terms of the Creative Commons Attribution Noncommercial License which permits any noncommercial use, distribution, and reproduction in any medium, provided the original author(s) and source are credited.

\section{References}

1. Attouch, H., Brezis, H. : Duality for the sum of convex funtions in general banach spaces. In: Barroso, J.A. (ed.) Aspects of Mathematics and its Applications, pp. 125-133. Elsevier Science Publishers, Amsterdam (1986)

2. Borwein, J. M.: Fifty years of maximal monotonicity. <http://www.carma.newcastle.edu.au/ jb616/fifty. pdf $>$

3. Boţ, R.I., Csetnek, E.R., Wanka, G.: A new condition for maximal monotonicity via representative functions. Nonlinear Anal. 67, 2390-2402 (2007)

4. Boţ, R.I., Grad, S.-M., Wanka, G.: Maximal monotonicity for the precomposition with a linear operator. SIAM J. Optim. 17, 1239-1252 (2006)

5. Burachik, R.S., Svaiter, B.F.: Maximal monotonicity, conjugation and the duality product. Proc. Amer. Math. Soc. 131, 2379-2383 (2003)

6. Fitzpatrick, S.: Representing monotone operators by convex functions, Workshop/ Miniconference on Functional Analysis and Optimization. In: Proceedings Centre Mathematical Analysis Australian National University, vol 20, pp. 59-65. Australian National University, Canberra (1988)

7. Martínez-Legaz, J.-E.: Some generalizations of Rockafellar's surjectivity theorem. Pac. J. of Optim. 4, 527-535 (2008)

8. Martínez-Legaz, J.-E., Théra, M.: A convex representation of maximal monotone operators. J. Nonlinear Convex Anal. 2, 243-247 (2001)

9. Penot, J.-P.: The relevance of convex analysis for the study of monotonicity. Nonlinear Anal. 58, 855-871 (2004)

10. Penot, J.-P., Zălinescu, C.: Some problems about the representation of monotone operators by convex functions. Anziam J. 47, 1-20 (2005)

11. Rockafellar, R.T.: Extension of Fenchel's duality theorem for convex functions. Duke Math. J. 33, 81-89 (1966)

12. Rockafellar, R.T.: On the maximality of sums of nonlinear monotone operators. Trans. Am. Math. Soc. 149, 75-88 (1970)

13. Simons, S.: From Hahn-Banach to Monotonicity, Lecture Notes in Mathematics. Vol. 1693, 2nd edn. Springer, Berlin (2008)

14. Simons S.: Banach SSD spaces and classes of monotone sets. http://arxiv/org/abs/0908.0383v2, posted August 26 (2009)

15. Simons, S., Zălinescu, C.: Fenchel duality, fitzpatrick functions and maximal monotonicity. J. of Nonlinear and Convex Anal. 6, 1-22 (2005)

16. Voisei, M.D., Zălinescu C.: Strongly-representable operators. J. Convex Anal. 16(3) (2009)

17. Zălinescu, C.: Convex Analysis in General Vector Spaces. World Scientific, Singapore (2002)

18. Zălinescu, C.: A new convexity property for monotone operators. J. Convex Anal. 13, 883-887 (2006)

19. Zeidler, E.: Nonlinear Functional Analysis and its Applications, II/B, Nonlinear Monotone Operators. Springer, New York (1990) 